\title{
Freeze-Dried Human Platelet-Rich Plasma Retains Activation and Growth Factor Expression after an Eight-Week Preservation Period
}

\author{
Yasuhiro Shiga ${ }^{{ }^{*}}$, Go Kubota ${ }^{{ }^{*}}$, Sumihisa Orita ${ }^{1}, \operatorname{Kazuhide~Inage~}^{1}$, Hiroto Kamoda ${ }^{1}$, Masaomi Yamashita ${ }^{1}$, \\ Toru Iseki ${ }^{2}$, Michihiro Ito ${ }^{2}$, Kazuyo Yamauchi ${ }^{1}$, Yawara Eguchi ${ }^{1}$, Takeshi Sainoh ${ }^{1}$, Jun Sato ${ }^{1}$, \\ Kazuki Fujimoto ${ }^{1}$, Koki Abe ${ }^{1}$, Hirohito Kanamoto ${ }^{1}$, Masahiro Inoue ${ }^{1}$, Hideyuki Kinoshita ${ }^{1}$, \\ Takeo Furuya ${ }^{1}$, Masao Koda ${ }^{1}$, Yasuchika Aoki ${ }^{3}$, Tomoaki Toyone ${ }^{4}$, Kazuhisa Takahashi ${ }^{1}$, Seiji Ohtori ${ }^{1}$
${ }^{1}$ Department of Orthopaedic Surgery, Graduate School of Medicine, Chiba University, Chiba, Japan
${ }^{2}$ Department of Blood Transfusion, Graduate School of Medicine, Chiba University, Chiba, Japan
${ }^{3}$ Department of Orthopaedic Surgery, Eastern Chiba Medical Center, Chiba, Japan
${ }^{4}$ Department of Orthopaedic Surgery, Showa University, Tokyo, Japan

\begin{abstract}
Study Design: Controlled laboratory study.
Purpose: This study aimed to evaluate the efficacy of platelet-rich plasma (PRP) stored at room temperature (RT), frozen, or after freeze-drying.

Overview of Literature: PRP enriches tissue repair and regeneration, and is a novel treatment option for musculoskeletal pathologies. However, whether biological activity is preserved during PRP storage remains uncertain.

Methods: PRP was prepared from blood of 12 healthy human volunteers ( $200 \mathrm{~mL} /$ person) and stored using three methods: PRP was stored at RT with shaking, PRP was frozen and stored at $-80^{\circ} \mathrm{C}$, or PRP was freeze-dried and stored at RT. Platelet counts and growth factor content were examined immediately after preparation, as well as 2, 4, and 8 weeks after storage. Platelet activation rate was quantified by flow cytometry.

Results: Platelet counts were impossible to determine in many RT samples after 2 weeks, but they remained at constant levels in frozen and freeze-dried samples, even after 8 weeks of storage. Flow cytometry showed approximately $80 \%$ activation of the platelets regardless of storage conditions. Almost no growth factors were detected in the RT samples after 8 weeks, while low but significant expression was detected in the frozen and freeze-dried PRP. Over time, the mean relative concentrations of various growth factors decreased significantly or disappeared in the RT group. In the frozen group, levels were maintained for 4 weeks, but decreased significantly by 8 weeks $(p<0.05)$. The freeze-dried group maintained baseline levels of growth factors for the entire 8 -week duration. Conclusions: Freeze-drying enables PRP storage while maintaining bioactivity and efficacy for extended periods.
\end{abstract}

Keywords: Platelet-rich plasma; Freeze drying; Blood preservation; Growth factors

Received Aug 12, 2016; Revised Sep 28, 2016; Accepted Nov 2, 2016

Corresponding author: Yasuhiro Shiga

Department of Orthopaedic Surgery, Graduate School of Medicine, Chiba University, 1-8-1 Inohana, Chuo-ku, Chiba 260-8670, Japan Tel: +81-43-226-2117, Fax: +81-43-226-2116, E-mail: yshiga1111@yahoo.co.jp

*These authors contributed equally to this work. 


\section{Introduction}

Platelet-rich plasma (PRP) is isolated from a patient's blood via centrifugation. The sequestration and concentration of platelets generate clinically useful levels of various growth factors such as platelet-derived growth factor (PDGF), insulin-like growth factor, and transforming growth factor- $\beta$ (TGF- $\beta$ ). In clinical use, PRP enhances neoangiogenesis, tissue repair, and regeneration [1], and is emerging as a novel treatment for musculoskeletal pathologies, such as ligament injury, muscle tears, tendinopathies, and osteoarthritis [2-5].

Kamoda et al. [6,7] reported that PRP accelerates bone union in a rat model of spinal posterolateral fusion, indicating that it is useful in orthopedic tissue reconstruction or fusion surgery, with some limitations. First, the patient's blood must be collected immediately before surgery to maintain its efficacy and invasiveness. Second, the extracted PRP must be activated during surgery, which requires an undesirable interruption in the procedure. Therefore, isolating and preserving PRP before surgery would be ideal. However, it is unknown whether preserved PRP is therapeutically effective. Some studies have indicated the possibility of freeze-drying PRP to maintain useful growth factor levels in the supernatant [8-10], although the biological effectiveness of freeze-dried PRP is unclear. Therefore, we investigated the efficacy of PRP samples that were preserved via either freezing or freezedrying by measuring their growth factor levels.

\section{Materials and Methods}

This study design was approved by our institution's ethics review committee, and all participants provided their written informed consent. We obtained PRP by collecting blood samples from 12 healthy volunteers $(200 \mathrm{~mL} /$ person) and stored the samples using three methods: (1) PRP was stored at room temperature (RT) with shaking, (2) PRP was frozen and stored at $-80^{\circ} \mathrm{C}$, or (3) PRP was freeze-dried and stored at RT. Platelet counts and growth factor levels were measured immediately after preparation, as well as after 2, 4, and 8 weeks of storage. Platelet activation rates were measured by flow cytometry.

\section{PRP preparation}

Blood samples (200 mL/person) were obtained using triple blood transfusion bags. After centrifugation (CR7BS, Hitachi, Tokyo, Japan) at $800 \times$ g for 5 minutes at RT, the supernatants were harvested (Fig. 1). Supernatant separations were then performed manually and visually using an isolation stand (Nippon-Flex, Tokyo, Japan). After a second centrifugation step (580×g for 20 minutes at RT), the final supernatant was isolated as fresh PRP. Each 200 $\mathrm{mL}$ sample of whole blood yielded approximately 10-15 $\mathrm{mL}$ of PRP, and $1 \mathrm{~mL}$ aliquots were prepared for the three groups of experimental samples.

\section{Freeze-dried PRP preparation}

Each of the PRP aliquots was weighed prior to freeze-drying. The test tubes were then rotated in an ethanol bath at $-60^{\circ} \mathrm{C}$ for membranous freezing (preliminary freezing), and then immediately frozen at $-30^{\circ} \mathrm{C}$ for 4 hours. The tubes were then attached to a vacuum freeze-dryer to complete the process. The freeze-dried PRP samples were
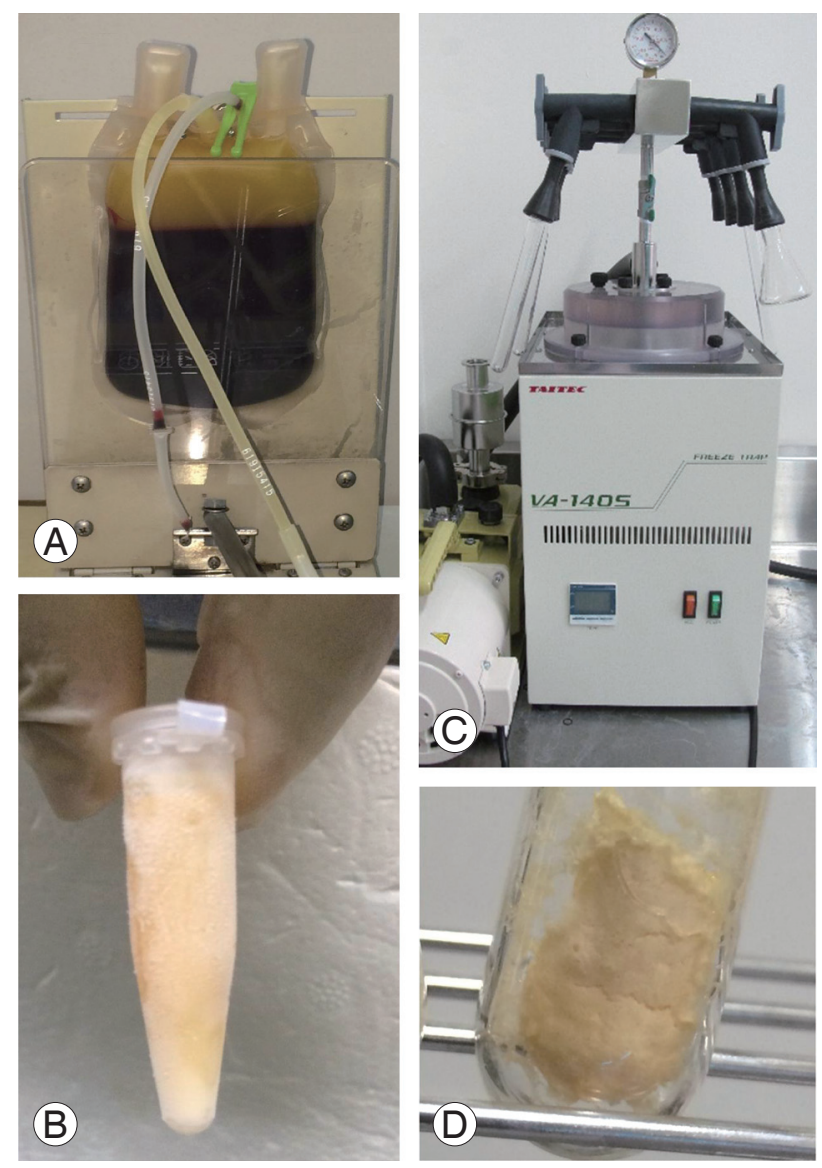

Fig. 1. Platelet-rich plasma (PRP) preparation. PRP was isolated as a supernatant from centrifuged blood $(\mathbf{A})$, frozen at $-80^{\circ} \mathrm{C}(\mathbf{B})$, and freeze-dried (C, D). 
resuspended in distilled water prior to assessment. To avoid any changes in component concentrations, the postresuspension weight was matched to the weight prior to freeze-drying.

\section{Platelet counts}

Platelets were counted using a K-4500 counter (Sysmex, Tokyo, Japan). To avoid deviations due to platelet aggregation, platelet counting was performed using frozen and lyophilized samples after thawing at RT and after 3-4 hours of resuspension in distilled water, respectively.

\section{Platelet activation}

PRP generally requires activation before use. Therefore, we activated platelets by adding $100 \mu \mathrm{L}$ of calcium chloride solution $(1 \mathrm{mEq} / \mathrm{mL}$, Otsuka Pharmaceutical, Tokyo, Japan) and $100 \mu \mathrm{L}$ of thrombin solution (Liquid Thrombin Mochida Softbottle 10,000, Mochida Pharmaceutical, Tokyo, Japan) to each milliliter of the PRP sample.

\section{Assessment of platelet activation via flow cytometry}

Flow cytometry was performed using a FACSCalibur instrument (BD Biosciences, East Rutherford, NJ, USA). Fresh PRP (before the activation), activated fresh PRP, and activated freeze-dried PRP (at 8 weeks after the preparation) were each mixed with anti-CD41a (platelet marker) and anti-CD62P (activated platelet marker) antibodies, and the percentages of activated platelets were assessed. According to the protocol, $1 \mathrm{~mL}$ of $1 \%$ paraformaldehyde in phosphate buffered saline was added to $10 \mu \mathrm{L}$ of each sample and allowed to stand for 30 minutes. Subsequently, both antibodies were incubated with each sample for 20 minutes, after which the samples were analyzed by flow cytometry.

\section{Growth factor detection and evaluation}

We quantified the expression levels of PDGF, TGF- $\beta$, vascular endothelial growth factor (VEGF), and epidermal growth factor (EGF), which are all tissue repair-associated growth factors present in PRP, using a Growth Factor Human Membrane Antibody Array (ab134002, Abcam, Cambridge, UK). Quantitative analysis of the membranous spots was performed with C-Digit Image Studio soft- ware (LI-COR, Lincoln, NE, USA). Due to the variations in the background concentration of each membrane, the concentration of each growth factor spot was calculated relative to a control spot on the same membrane. Growth factor levels were analyzed immediately after preparation, as well as 2,4 , and 8 weeks afterward.

\section{Statistical analysis}

Platelet counts and the relative concentration of each growth factor are expressed as mean \pm standard deviation. All data were analyzed by Mann-Whitney U test. A pvalues of $<0.05$ were considered statistically significant.

\section{Results}

\section{Platelet counts}

The mean platelet counts were $(31.5 \pm 7.3) \times 10^{4} / \mu \mathrm{L}$ in the whole blood samples and $(130.1 \pm 41.1) \times 10^{4} / \mu \mathrm{L}$ in the PRP samples. The platelet counts in the PRP samples were approximately 4.1 -fold higher than those in peripheral blood samples, and all samples were concentrated evenly. Storage at RT generated turbidity and sedimentation of crystals over time, which made it impossible to obtain platelet counts in many RT samples after 2 weeks. The platelet counts remained relatively constant in the frozen and freeze-dried samples, even after 8 weeks of preservation (Fig. 2).

\section{Platelet activation and growth factor levels}

Flow cytometry revealed approximately $80 \%$ platelet activation, regardless of storage conditions (Fig. 3).

We tested the fresh PRP samples for various growth factors, which included PDGF (AA, AB, and BB), VEGF, TGF- $\beta$, and EGF (Fig. 4A). At the end of the 8-week observation period, almost no growth factors were detected in RT samples (Fig. 4B), while low but significant levels of expression were detected in the frozen PRP samples (Fig. 4C). In the freeze-dried PRP, some growth factors exhibited slightly reduced levels, but the expression levels were comparable to those of fresh PRP (Fig. 4D).

Fig. 5 shows the changes in the mean relative concentration of each growth factor over time. In the RT samples, a clear reduction in growth factor expression occurred after 2 weeks. In the frozen samples, expression levels were 


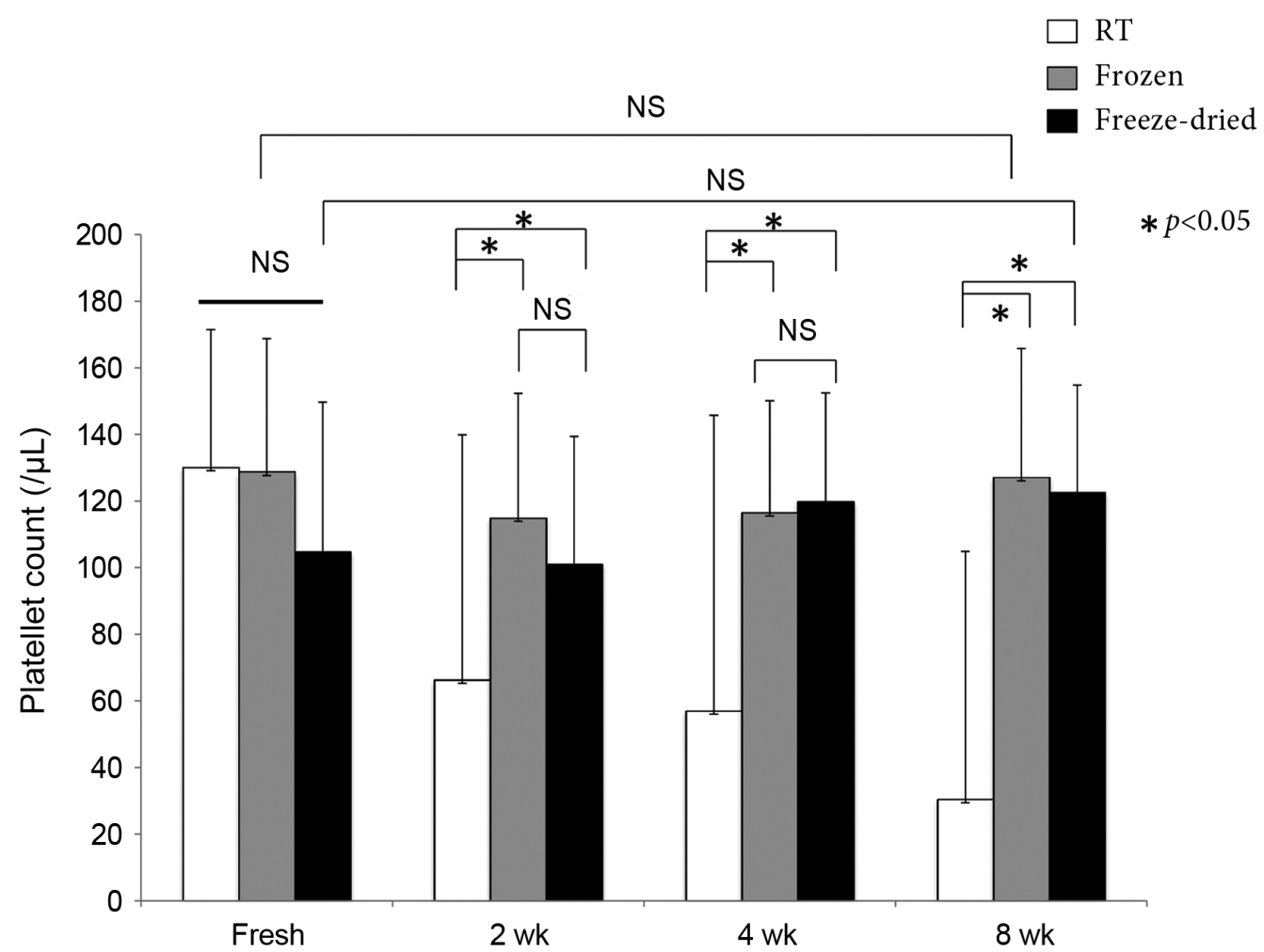

Fig. 2. Platelet counts in platelet-rich plasma (PRP). PRP that was preserved at room temperature (RT) exhibited loss of platelets, although the frozen and freeze-dried samples maintained their platelet counts throughout the 8-week duration of the experiment. NS, not significant.
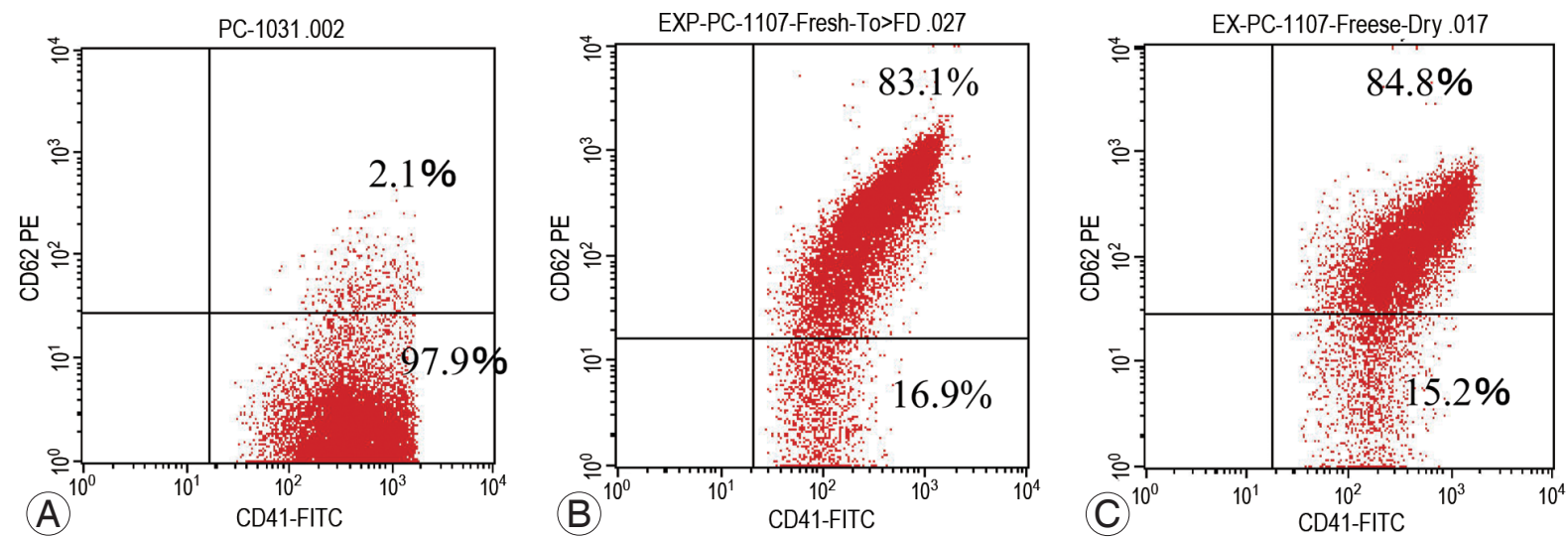

Fig. 3. Platelet activation rates. (A) Fresh platelet-rich plasma (PRP) immediately after preparation (pre-activation). (B) Activated fresh PRP. (C) Activated freeze-dried PRP 8 weeks after preparation. The percentages of activated platelets in the freeze-dried samples were similar to those in the fresh PRP, even after 8 weeks. Horizontal axis, anti-CD41a antibody (platelet marker); vertical axis, anti-CD62P antibody (activated platelet marker).

maintained at 4 weeks. However, expression of TGF- $\beta$, vascular endothelial growth factor receptor 2, and EGF decreased by 8 weeks $(p<0.05)$. In contrast, the expression levels of growth factors in the freeze-dried samples were maintained throughout the 8-week duration of the experiment.

\section{Discussion}

In the present study, we examined the biological activ- 

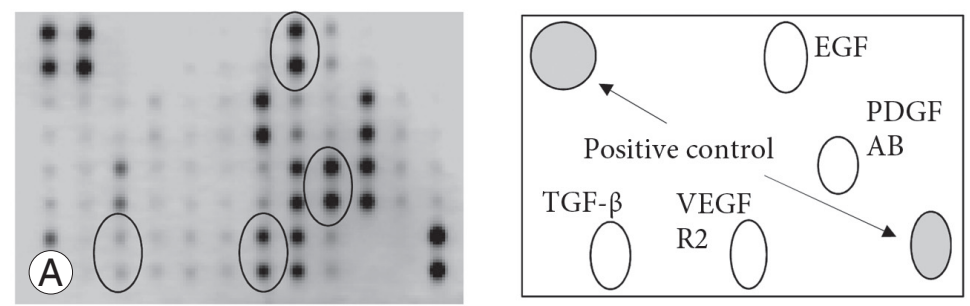

(scheme)
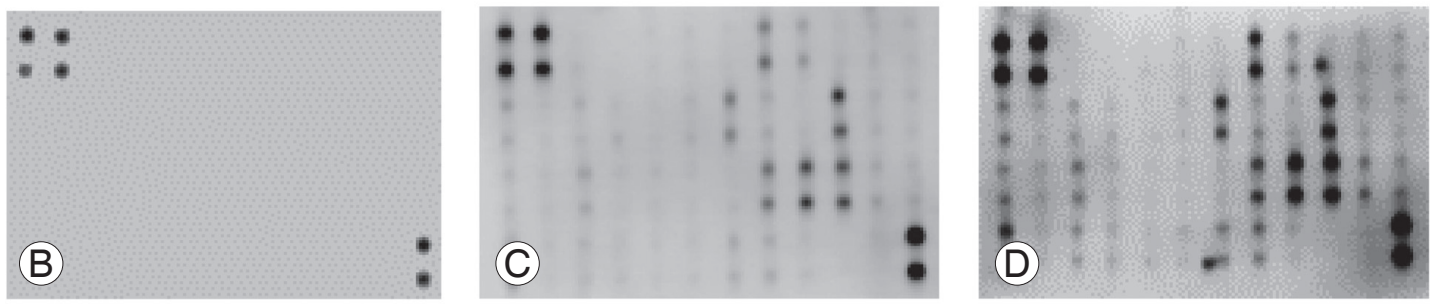

Fig. 4. Detection of growth factors. (A) Expression levels of platelet-derived growth factor $A B(P D G F A B)$, vascular endothelial growth factor receptor 2 (VEGF R2), transforming growth factor- $\beta$ (TGF- $\beta$ ), and epidermal growth factor (EGF) were evaluated in fresh platelet-rich plasma (PRP) according to the intensity of the spots. The location of each is shown in the scheme. (B) PRP was stored at room tempertature for 8 weeks, and all growth factor expressions were almost undetectable. (C) Frozen PRP was stored for 8 weeks, after which faint expression of growth factors was observed. (D) The freeze-dried PRP was stored for 8 weeks, and almost all growth factors were expressed robustly.

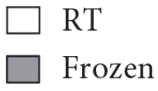

Freeze-dried

PDGF AB

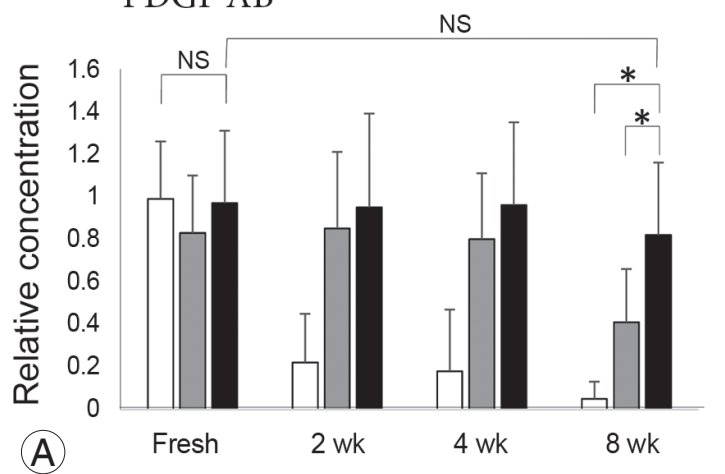

VEGF R2

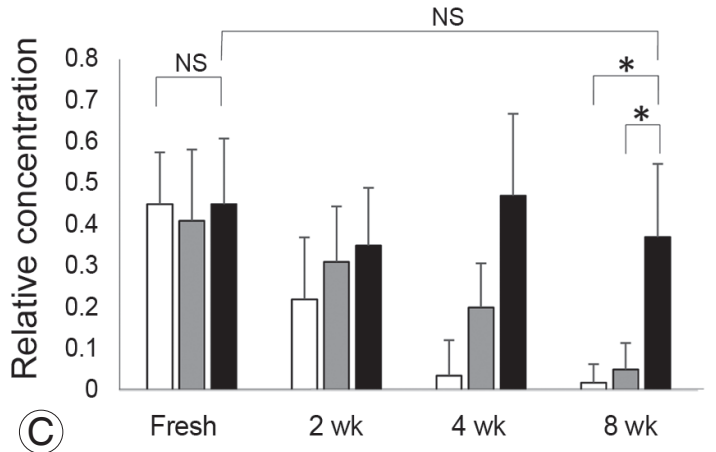

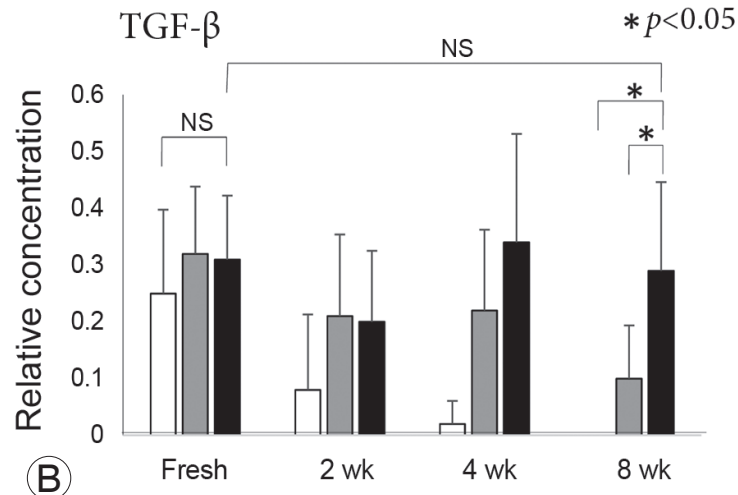

EGF

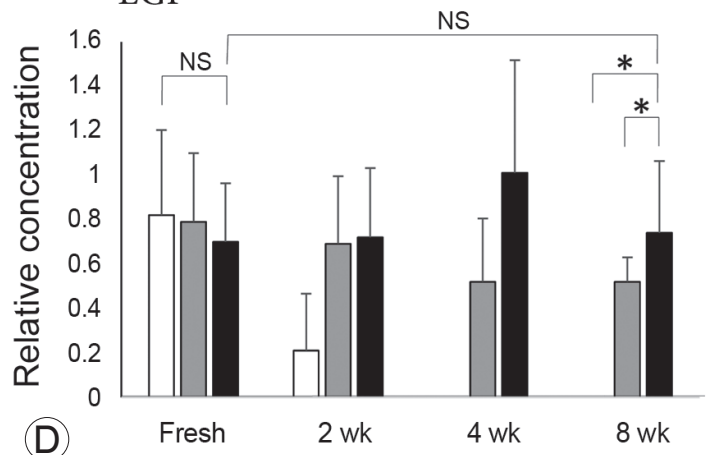

Fig. 5. Relative concentration of each growth factor (fold increase). Platelet-derived growth factor AB (PDGF AB) (A), transforming growth factor- $\beta$ (TGF- $\beta$ ) (B), vascular endothelial growth factor receptor 2 (VEGF R2) (C), and epidermal growth factor (EGF) levels (D) were assessed over time. In the room temperature (RT) group, there was a clear reduction and/or disappearance of the growth factor expression after 2 weeks. In the frozen group, growth factor expression levels were decreased at 8 weeks. In contrast, the freezedried group maintained its original expression levels for the 8-week duration of the study. NS, not significant. 
ity of PRP stored at RT, frozen, or after freeze-drying. We found that, while RT storage significantly reduced platelet counts, platelets persisted for 8 weeks in the frozen or freeze-dried samples. Platelet activation was also maintained when activated PRP was freeze-dried. The levels of growth factors in the RT samples gradually and significantly decreased over time, although these levels were maintained in the freeze-dried samples. Unlike frozen PRP, freeze-dried PRP maintained levels of growth factors that were comparable to the levels in fresh PRP. These results suggest that pre-prepared PRP retains its efficacy when it is appropriately freeze-dried for storage. PRP is generally stored in a freezer and thawed before use. However, the effect of thawing on the biological activity of PRP has not been fully investigated. Freezing is a common method of cell storage, although it can cause cold-shock damage $[11,12]$. Therefore, freeze-drying platelets, which was first proposed by Wolkers et al. [13] in their earlier study, is an alternative preservation technique.

Pietramaggiori et al. $[14,15]$ reported the bioactivity of growth factors and their healing effects in freeze-dried PRP that was freshly reconstituted for use in chronic wounds. Nakajima et al. [9] also reported bioactivity in freeze-dried PRP immediately after reconstitution into a form adsorbed to a biodegradable polymer material, while Horimizu et al. [8] reported that the storage of freeze-dried PRP-coated collagen sponges at $4^{\circ} \mathrm{C}$ did not cause a significant loss in bioactivity. In the present study, we analyzed freeze-dried PRP that had not otherwise been treated, as we did not want any adverse reactions or infections that might be caused by foreign materials to confound our results. There are currently no studies on freeze-dried PRP preserved for 8 weeks after preparation with no further treatments. Flow cytometry revealed that platelet activation was maintained in freeze-dried PRP. Any such activation did not appear to be destructive.

Growth factors are vital for platelet efficacy in various applications. In the present study, growth factor levels were preserved for 8 weeks after freeze-drying. In contrast, freezing alone produced inferior results at 8 weeks, which suggests that freezing alone is not suitable for longterm storage, although long-term preservation of frozen PRP is commonly performed in the clinical setting. The growth factors that we evaluated function in tissue repair and bone fusion $[2,16]$. PDGF enhances collagen synthesis and bone cell proliferation $[17,18]$, TGF- $\beta$ enhances the proliferative activity of fibroblasts and stimulates the biosynthesis of type I collagen and fibronectin [19,20], VEGF induces angiogenesis via migrating endothelial cells [21], and EGF orchestrates cellular proliferation and differentiation of epithelial cells [22].

The results of our study suggest that freeze-drying is the most suitable technique for preserving PRP bioactivity, as it preserves both platelet counts and growth factor levels, while freezing only maintains the platelet counts. In this context, long-term cryopreservation may adversely affect the levels of growth factors owing to continuous exposure to low temperature rather than the freezing procedure itself. Therefore, further studies that include in vivo experiments are required to measure the efficacy of freezedried PRP in enhancing tissue repair or bone healing and to verify that freeze-dried PRP has the same safety profile as that of fresh PRP. Furthermore, these studies should carefully assess the risk of complications, such as infection, excessive inflammatory reactions (e.g., graft-versushost disease), and ectopic ossification. In a previous study, the use of fresh PRP was shown to cause no problems in the clinical setting. Freeze-dried PRP should be prepared in a clean room using sterile procedures in order to avoid infections. Once the feasibility of sterile preparation and the safety of freeze-dried PRP have been confirmed, this product can be used for various orthopedic indications, such as treatment of emergency injury or during surgeries, in a form that is similar to blood transfusion preparations and biological agents. Another benefit of freeze-dried PRP is easy storage, as its powder form enables storage in a refrigerator or even at RT. This would be very convenient for mixing with bone grafts, placement within the substantia spongiosa of transplanted bones, or incorporation into artificial bones after dissolving in water. Powdered PRP may also facilitate the controlled release of active growth factors, as PRP concentrations can be adjusted during the dissolving process. Thus, we expect that freeze-dried PRP has broad applications in the tissue repair and orthopedic fields, as it provides a safer alternative to other biological products.

The present study has several limitations. First, we did not assess platelet morphology. Further histological studies, such as electron microscopy, should be performed to confirm that the appropriate morphology is preserved. Second, this was a preliminary study with a small sample size; therefore, further studies with larger samples are required to validate our findings. Third, the freeze-dried PRP was prepared under non-sterile conditions. We aim 
to develop a method for sterile preparation in future studies. Finally, we did not evaluate the actual amounts of each growth factor in PRP, as we initially planned to evaluate the overall state of various growth factors in PRP using the Growth Factor Membrane Antibody Array followed by densitometry. More quantitative evaluation will be required in future studies to evaluate the actual concentrations of growth factors using more exact protein assays such as enzyme-linked immunosorbent assay.

We have already used fresh PRP in clinical practice, and have obtained good results with no major complications. In the future, we plan to examine the efficacy and safety of freeze-dried PRP using a rat posterolateral lumbar fusion model. A proposal for this research project has already been submitted to the ethics committee of our hospital. If the efficacy and safety of freeze-dried PRP is proven, its regular use will be possible.

\section{Conclusions}

In conclusion, our results suggest that freeze-dried human PRP maintains activated platelet counts and growth factor levels that are comparable to those of fresh PRP, even after 8 weeks of storage. These results highlight the potential clinical value of freeze-dried PRP.

\section{Conflict of Interest}

No potential conflict of interest relevant to this article was reported.

\section{References}

1. Anitua E, Andia I, Ardanza B, Nurden P, Nurden AT. Autologous platelets as a source of proteins for healing and tissue regeneration. Thromb Haemost 2004;91:4-15.

2. Alsousou J, Thompson M, Hulley P, Noble A, Willett $\mathrm{K}$. The biology of platelet-rich plasma and its application in trauma and orthopaedic surgery: a review of the literature. J Bone Joint Surg Br 2009;91:987-96.

3. MS AH, Mohamed Ali MR, Yusof A, George J, Lee LP. Platelet-rich plasma injections for the treatment of hamstring injuries: a randomized controlled trial. Am J Sports Med 2014;42:2410-8.

4. Moraes VY, Lenza M, Tamaoki MJ, Faloppa F, Belloti JC. Platelet-rich therapies for musculoskeletal soft tissue injuries. Cochrane Database Syst Rev 2014;(4): CD010071.

5. Tietze DC, Geissler K, Borchers J. The effects of platelet-rich plasma in the treatment of large-joint osteoarthritis: a systematic review. Phys Sportsmed 2014;42:27-37.

6. Kamoda H, Yamashita M, Ishikawa T, et al. Plateletrich plasma combined with hydroxyapatite for lumbar interbody fusion promoted bone formation and decreased an inflammatory pain neuropeptide in rats. Spine (Phila Pa 1976) 2012;37:1727-33.

7. Kamoda H, Ohtori S, Ishikawa T, et al. The effect of platelet-rich plasma on posterolateral lumbar fusion in a rat model. J Bone Joint Surg Am 2013;95:110916.

8. Horimizu M, Kawase T, Nakajima Y, et al. An improved freeze-dried PRP-coated biodegradable material suitable for connective tissue regenerative therapy. Cryobiology 2013;66:223-32.

9. Nakajima Y, Kawase T, Kobayashi M, Okuda K, Wolff LF, Yoshie H. Bioactivity of freeze-dried plateletrich plasma in an adsorbed form on a biodegradable polymer material. Platelets 2012;23:594-603.

10. Tablin F, Walker NJ, Hogle SE, Pratt SM, Norris JW. Assessment of platelet growth factors in supernatants from rehydrated freeze-dried equine platelets and their effects on fibroblasts in vitro. Am J Vet Res 2008;69:1512-9.

11. Balint B, Paunovic D, Vucetic D, et al. Controlledrate versus uncontrolled-rate freezing as predictors for platelet cryopreservation efficacy. Transfusion 2006;46:230-5.

12. Owens M, Cimino C, Donnelly J. Cryopreserved platelets have decreased adhesive capacity. Transfusion 1991;31:160-3.

13. Wolkers WF, Walker NJ, Tablin F, Crowe JH. Human platelets loaded with trehalose survive freeze-drying. Cryobiology 2001;42:79-87.

14. Pietramaggiori G, Kaipainen A, Czeczuga JM, Wagner CT, Orgill DP. Freeze-dried platelet-rich plasma shows beneficial healing properties in chronic wounds. Wound Repair Regen 2006;14:573-80.

15. Pietramaggiori G, Scherer SS, Mathews JC, et al. Healing modulation induced by freeze-dried plateletrich plasma and micronized allogenic dermis in a diabetic wound model. Wound Repair Regen 2008; 16:218-25. 
16. Foster TE, Puskas BL, Mandelbaum BR, Gerhardt MB, Rodeo SA. Platelet-rich plasma: from basic science to clinical applications. Am J Sports Med 2009;37:2259-72.

17. Raines EW, Ross R. Platelet-derived growth factor: I. High yield purification and evidence for multiple forms. J Biol Chem 1982;257:5154-60.

18. Shah P, Keppler L, Rutkowski J. A review of platelet derived growth factor playing pivotal role in bone regeneration. J Oral Implantol 2014;40:330-40.

19. Bonewald LF, Mundy GR. Role of transforming growth factor-beta in bone remodeling. Clin Orthop
Relat Res 1990;(250):261-76.

20. Celeste AJ, Iannazzi JA, Taylor RC, et al. Identification of transforming growth factor beta family members present in bone-inductive protein purified from bovine bone. Proc Natl Acad Sci U S A 1990;87:98437.

21. Ferrara N, Gerber HP. The role of vascular endothelial growth factor in angiogenesis. Acta Haematol 2001;106:148-56.

22. Herbst RS. Review of epidermal growth factor receptor biology. Int J Radiat Oncol Biol Phys 2004;59(2 Suppl):21-6. 